\title{
ECCENTRIC AND CONCENTRIC MOTION OF HAMSTRING DURING THE LEG CURL
}

\author{
Shiva Bikram Thapa, Suresh Basnet*, Raju Khanal \\ Central Department of Physics, Tribhuvan University, Kirtipur, Kathmandu 44613, Nepal \\ *Corresponding author: sbplasma1986@gmail.com, suresh.745711@cdp.tu.edu.np \\ (Received:July 04, 2021; Revised: December 7, 2021; Accepted: December 21, 2021)
}

\begin{abstract}
The force experienced by the hamstring during the leg curl has been numerically investigated using the conservation theorem. The center of the meniscus is assumed to be the pivot point along with the uniform distribution of forces in the frictionless environment. The variation of force experienced by the hamstring during the concentric motion of the leg curl has been derived and graphically illustrated. It is found that the force experienced by the hamstring increases with the increase in length of the lower leg and its weight as well. The magnitude of force decreases with the increase in distance from the pivot to insertion. However, the magnitude of force increases from about 3.60 to $4.79 \mathrm{kN}$ in the practically valid region 3 to $4 \mathrm{~cm}$ distance from the pivot to insertion with the increase in weight of lower leg from 5 to $15 \mathrm{~kg}$. On the other hand, the magnitude of force increases from about 3.75 to $9.80 \mathrm{kN}$ with the increase in weight suspended on the machine from 10 to $40 \mathrm{~kg}$. In addition, the force decreases with the increase in upper leg dimension, but it linearly increases with the increase in the angle of suspension.
\end{abstract}

Keywords: A weight suspended, athlete, hamstring muscle, isotonic contraction, lower leg, moment

\section{INTRODUCTION}

The responsive behavior of tension generating sites of muscle fibers under the action of externally applied force is known as muscle contraction and it can happen without the actual movement of the muscle (Faulkner, 2003). Muscle contraction is extensively studied based upon the variation of force and muscle length. The terms "eccentric" and "concentric" are concerned with the muscle contraction in the physiological science (Hill, 1925). Depending upon the nature of tension applied, the muscle contraction can be categorized in two types: isometric and isotonic contractions (Padulo et al., 2013). In the isometric muscle contraction, muscle length does not change during the movement of muscle. For example, plank, holding the lower leg at maximum height during leg curl for a second or few fractions of a second (Faulkner, 2003). While the isotonic muscle contraction requires the length of the muscle to shorten or lengthen under the action of externally applied tension. For example, biceps curl and push-up (Padulo et al., 2013). The isotonic muscle contractions further categorized in two types: concentric and eccentric contractions (Padulo et al., 2013). Muscle activation in which the length of the muscle contracts under the action of externally applied force is termed as concentric contraction. For example, contractions of hamstring muscle towards hip region during the upward motion of leg curl (Faulkner, 2003). Muscle activation in which the length of the muscle lengthens under the action of externally applied force is termed as eccentric contraction. For example, the hamstring muscle extends towards knee joint region during the downward motion of leg curl (Floyd \& Thompson, 2015). The study of muscle contraction has gained growing interest in diverse fields such as sports training, physical medicine and rehabilitation (Croisier et al., 2002; Kjaer \& Heinemeier, 2014; Vogt \& Hoppeler, 2014; Hody et al., 2019). The electrical activity of the muscles is experimentally shown to be linearly dependent upon the velocity of eccentric and concentric contractions (Bigland \& Lippold, 1954). As compared to other exercises, the concentric part of the muscle movement for leg curl is significant in the electromyography activity (Wright et al., 1999). Leg curl (hamstring curl) is an isolation workout which activates the muscle fibers primarily of hamstring muscle. This leg curl activates the three types of muscles: semitendinosus, semimembranosus and long head of biceps femoris constitute hamstring muscles.

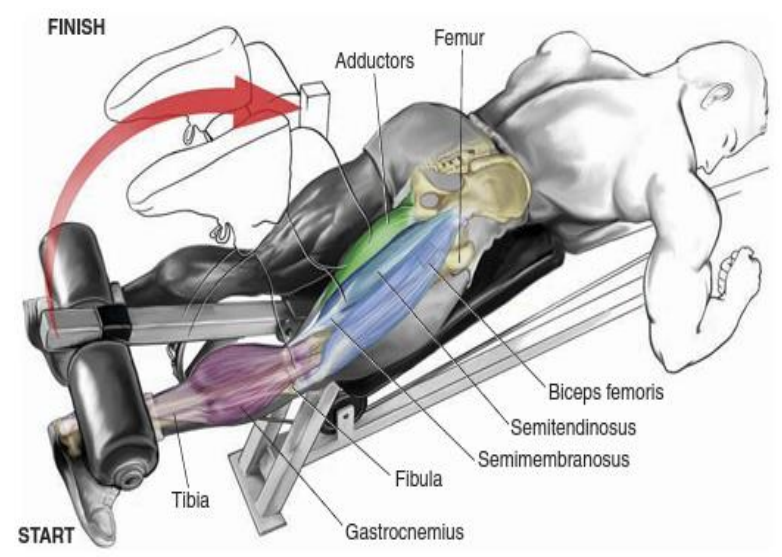

Figure 1. Schematic diagram of muscle contraction during leg curl. (Source: https:// robinsonsstrengthandendurancecoaching.wordpress.com /2018/01/12/leg-strength-training).

When we perform the leg curl, these three-muscle groups come together as one entity i.e., hamstring and help to lift our lower leg through the insertion slightly below knee (Aagaard et al., 1998). The athlete performing the leg curl has to lie down in prone position along the machine and lift the padded roller using their lower leg as shown in the Fig. 1. The athlete has to then lift the padded roller with the tendon and rotate the lower leg near the glutes by using hamstring. In that case, the distribution of force is 
assumed to be uniform and targets only hamstring muscle neglecting its effects on other muscles such as calves and glutes (Christ \& Knortz, 1981).An athlete performing leg curl has to remain static and maintain a firm grip of their hands on the handle bar of the machine provided (Christ \& Knortz, 1981). The study of muscle contraction has been carried out in the past which uses Newton's laws of motion under the linear and quadratic approximation of the medium resistance (Milošević, et al., 2000). An object rotating about a fixed axis carries certain angular momentum. Angular momentum is the product of object's inertia and the rotational velocity. During the rotational motion about any fixed axis of rotation, the angular momentum is always conserved (Ling et al., 2016). Muscle movements during exercise are studied to a great extent by using the conservation of angular momentum and work energy theorem. Mjolsness et al. (2004) presented an experiment for ten weeks to study the eccentric muscle contraction for hamstring curl and Nordic hamstring. They have used dynamometer to calculate the torque during hamstring curl and visual analog scale to measure the highest level of muscle soreness. It was found that the Nordic hamstring is more effective than that of hamstring curl for the eccentric contraction. Kilgallon et al. (2007) studied the relationship between torque and angle for hamstring over the period of three weeks using Con-Trex MJ dynamometer. It was found that the change in angle of peak isometric torque is significant for eccentric and concentric groups of adults.

In this work, we have studied the concentric motion of hamstring during upward motion and eccentric motion of hamstring during downward motion. However, the isometric motion is not studied in the present case. At the maximum height attained by the padded roller, an athlete can rest for few seconds which is the isometric motion for hamstring. But such mechanisms are hard to account for as there is no displacement of the system. It is found that the force experienced by athlete during the hamstring increases with the increase in length of lower leg and its weight. Although the magnitude of force decreases with the increase in distance between pivot and insertion, it increases with the increase in weight suspended on machine. Furthermore, the force linearly increases with the increase in angle of suspension; however, it decreases with the increases in upper leg dimension.

\section{MODEL DESCRIPTION AND BASIC EQUATIONS}

Conservation of angular momentum is written as, if the system is torque free, then its angular momentum is conserved. Torque free doesn't necessarily imply zero torque, but the net torque acting on the system is zero.

$$
\sum \tau_{0}=0
$$

where $\tau_{0}$ is the torque about the pivot point (Ling et al., 2016) and this equation (1) is a key mathematical expression to study the concentric and eccentric muscle activation in the human body. According to work energy theorem, the work is done by the sum of all forces acting on a particle equals the change in kinetic energy of the particle. If the system moves under the action of a force from any arbitrary point $A$ to point $B$, then the work done is given by,

Work done $=\frac{1}{2} m\left(v_{A}^{2}-v_{B}^{2}\right)$

where the terms appearing in equation (2) are defined as $v_{A}$ and $v_{B}$ are the velocities of the particles at points $A$ and $B$ respectively and $m$ is the mass of respective particle. Muscle contraction in human body under the action of externally applied force usually involves movement of muscle about certain pivot point. For example, while working with leg curl it would be much easier to take the center of the meniscus at the knee joint as the pivot point. For simplicity, we would not take kinetic as well as static frictions present in the knee joint. In a study of ten cadaver knees, it was found that the mean distance between tibial plateau and the flexor tendons is $41.0 \pm 4.6 \mathrm{~mm}$ (Grassi et al., 2013). This distance is loosely equal to the insertion of hamstring around tendon. Hence, it helps us to discriminate the feasibility of theoretically obtained values in our work.

The arrangement of lower leg in prone position along with the padded roller at the lower end of the tendon in lower leg constitutes a lever and its schematic diagram is shown in Fig. 2. Suppose $W_{\mathrm{s}}$ and $W_{l}$ are the weight suspended on the machine and weight of the lower leg, respectively. The point $O$ is the pivot about which the lever rotates and is so taken that it lies at the center of the meniscus at the knee joint. Initially, the lever is rested at an angle $\theta$ with the negative $\mathrm{Y}$-axis, and it is rotated by an angle $a$ and brought back to its initial position which completes one repetition of leg curl. Theoretically the angle $\theta$ can have any possible values; however, leg curl machine is created in such a way that an athlete performing the leg curl simply cannot lower the value of $\theta$ to 0 . The padded roller can only be suspended to certain minimum angle and therefore the work done is only significant to certain angular range of $\alpha$. Suppose $\overrightarrow{\boldsymbol{F}}$ is the force experienced by the hamstring muscle while pulling the lever, which makes an angle $\phi$ with the line of action of the lower leg. The hamstring is wrapped around the knee joint in such a way that it lies at a distance $x_{3}$ from the pivot point $O$. The other various terms used in the free body diagram are as follows: $L$ is the length of the lower leg from foot to knee joint, $x_{1}$ is the perpendicular distance from the line of action of center of mass of the lower leg to the line of action of pivot point, $x_{2}$ is the perpendicular distance from the line of action of center of mass of the padded roller to the line of action of pivot point and $r$ is the distance from pivot point to the center of mass of weight suspended.

The term $x_{3}$ is the distance between the pivot point $O$ to the insertion of hamstring muscle, which lies slightly below the point $O$. The entire lower leg is rotated about the pivot point $O$. However, the hamstring muscle is wrapped around the tendon in such a way that it lies slightly below the pivot point $O$. As a result, the components of force 
generated must be taken into account from the insertion point below the pivot point $O$. This is also the point about which the hamstring pulls the entire lower leg weight.

From free body diagram (Fig. 2), we can write,

$$
x_{1} W_{l}+x_{2} W_{s}=x_{3} F \sin \phi
$$

Using equation (3), the work done due to torque is given by,

$$
W=\int_{0}^{\alpha} \frac{x_{1} W_{l}+x_{2} W_{s}}{x_{3} \sin \phi} r d \alpha
$$

Here the value of $\theta$ and $\phi$ is known at the beginning of the exercise and the force term in the integral is independent of $\alpha$ and equation (4) yields;

$$
W=\frac{x_{1} W_{l}+x_{2} W_{s}}{x_{3} \sin \phi} r \alpha
$$

Equation (5) is our required expression for work done by hamstring during concentric motion of leg curl. The critical assumption that we have made in deriving this formula is the angle $\phi$ remains constant throughout the exercise because this angle changes by a very small factor. The schematic diagram for the calculation of work done by hamstring during the downward motion is shown in the Fig. 3. The total mass of the system (padded roller and leg) finally comes to rest at the maximum height point $A$ after the completion of its upward motion i.e., concentric motion.

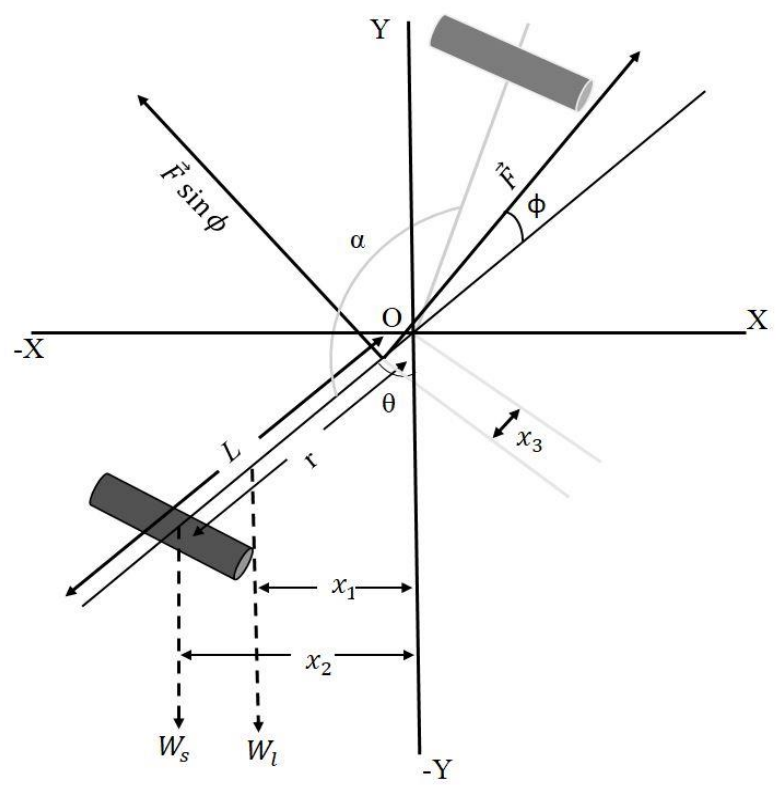

Figure 2. Schematic representation of free body diagram for the leg curl.

All the system's energy is converted into potential energy at the maximum height $A$. The system is at height $b$ with respect to point $B$ which is taken as ground reference. The padded roller immediately experiences gravity and comes into motion, but an athlete has to do some work in the opposite direction to overcome gravity in order to maintain a controlled eccentric motion.

Therefore, the total energy of the system at height $b$ after the completion of its upward motion is given by,
Total energy at point $A=M g h$

where $M$ is the mass of the system and $g$ is the acceleration due to gravity. If the system only moves under the influence of gravity, the total energy of the system during its downward motion just before coming to rest is given by,

Total energy at point $B=\frac{1}{2} M v^{2}$

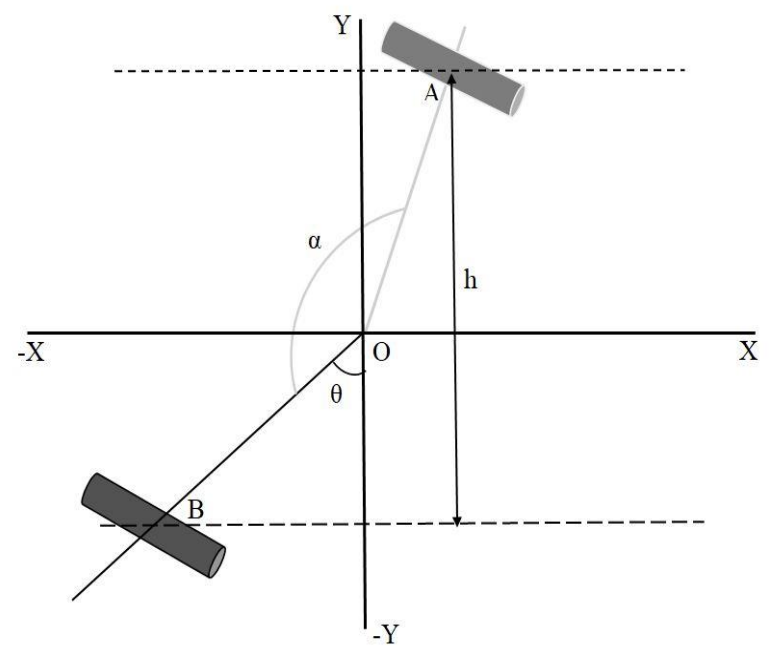

Figure 3. Schematic representation of eccentric motion of hamstring during the leg curl.

Assuming that the reference point $B$ is the ground, the conservation of energy from equations (6) and (7) yields;

$$
v=\sqrt{2 g h}
$$

Here equation (8) gives the velocity $(v)$ of the system before comes to the rest. However, while performing the leg curl if an athlete permits the system to fall only under the action of gravity, the fall would be abrupt and sudden. Hence, in order to keep the downward motion of the system stable, athletes must perform certain work in the opposite direction of gravity. The quantity that can be controlled here is acceleration, which is calculated at point $A$ as

$$
\text { Acceleration }=\frac{v_{B}}{t}
$$

where $t$ is the time taken by the system of padded roller and leg to complete its downward motion from the maximum height. Using equations (6) and (9), the work done by the athlete against the gravity to keep the system stable is given by,

$$
\text { Work done }=M\left(g-\frac{v_{B}}{t}\right) h
$$

Therefore, the total work done [sum of equations (5) and (10)] for $n$ number of repetitions by athlete during the upward and downward motion of leg curl is

$$
W=n\left[\frac{x_{1} W_{l}+x_{2} W_{s}}{x_{3} \sin \phi} r \alpha+M\left(g-\frac{v_{B}}{t}\right) h\right]
$$


The first term in equation (11) is due to contribution of concentric motion whereas; the second term is due to the contribution of eccentric motion. The variation of force experienced by hamstring muscle for people of different heights can be studied by expressing distances in terms of length of lower leg $L$. The schematic diagram for the calculation of force in terms of length of lower leg is shown in Fig. 4. The weight of the lower leg lies at the center of gravity of lower leg which is at distance $L / 2$ from the pivot $O$. For the simplicity, the distance from pivot to the point where padded roller is placed is taken to be $7 \mathrm{~L} / 8$.

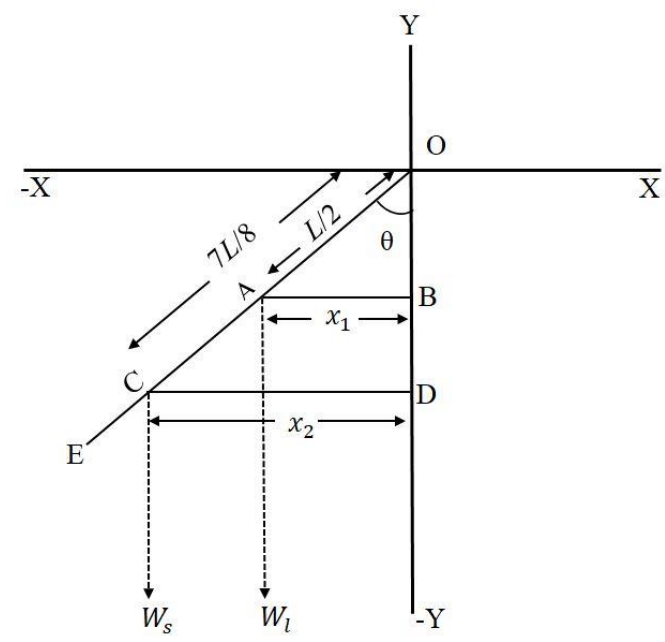

Figure 4. Schematic diagram for expressing distances $x_{1}$ and $x_{2}$ in terms of length of lower leg $L$.

Using Pythagoras theorem in $\triangle \mathrm{AOB}$,

$$
x_{1}=\left(\frac{L}{2}\right) \sin \theta
$$

Similarly,

$$
x_{2}=\left(\frac{7 L}{8}\right) \sin \theta
$$

Above equations (12) and (13) are vital to understand the nature of force during concentric motion for people of different heights.

$$
F=\frac{\left(W_{l} / 2+7 W_{s} / 8\right) L \sin \theta}{x_{3} \sin \phi}
$$

The above equation (14) is the force experienced by hamstring during upward motion of the system i.e., concentric motion. The equation (5) is re-written in terms of length of lower leg $(L)$ so that the variation of force can be studied for large group of people. The study of muscle contraction of hamstring during leg curl is very important for athletes as these studies help to prevent any injury on the hamstring muscle. The force on the hamstring muscle during leg curl depends upon the external factors such as weight suspended on the machine as well as the internal factors such as insertion around tendon and the dimensions of upper leg. This also implies that even when other quantities are kept constant, the force can vary from person to person on smaller scale.

\section{RESULTS AND DISCUSSION}

In the present work, we study the variation of force with various parameters such as weight of the lower leg, weight suspended on the machine, length of lower leg, insertion of hamstring muscle and dimension of the upper leg. The force experienced by hamstring muscle during concentric motion of leg curl as the function of length of the lower leg and weight of lower leg at constant weight suspended on machine $W_{\mathrm{s}}=15 \mathrm{~kg}$ is shown in the Fig. 5(a). The force experienced by the hamstring muscle is found to increase linearly with increase in length of lower leg as well as weight of the lower leg. The increment of force with the increase in length of lower leg is qualitatively similar with the previous work (Maganaris, 2001). With the increase in weight of lower leg, the increment rate of force during hamstring curl gets increased. The force gets increased with the increase in length of lower leg for the constant weight of lower leg. This suggests that for constant weight suspended on the machine, the tall people will experience more force than the short people because of tall people have longer lower leg length. When length of lower leg is increased, the distance from negative $y$-axis to the center of mass of lower leg and the padded roller is also increased. As a result, moment arm about center of meniscus increases which causes to increase the magnitude of force linearly. As the weight of lower leg increases, the hamstring has to apply more force in the upward direction to lift the system in that direction, as a result the force on the hamstring also increases.

The force experienced by the hamstring muscle during the concentric motion of the leg curl depends on the weight of lower leg and insertion around tendon of the athletes and the graphical illustration for the different weights of lower leg is depicted in Fig. 5(b). It is found that the force decreases with the increase in distance between the pivot and insertion for all the cases, while the magnitude of force increases with the increase in weight of lower leg. The force experienced by the hamstring at insertion lies in between 1 to $3 \mathrm{~cm}$ from pivot is found to be higher which is greater than $3.60 \mathrm{kN}$. This magnitude of force is only suitable for the theoretical study of insertion around tendon as it significantly larger. This region (less than 3 $\mathrm{cm}$ ) is referred as practically discrepancy region as it is impossible to measure such a discrepancy in force for different insertions. The region clearly depicts that the insertion cannot lie very close to the pivot. However, the practically valid insertion is around 3 to $4 \mathrm{~cm}$ and that can only vary for different persons on a smaller scale such as one tenth or one hundredth of a centimeter (Grassi et al., 2013). With the increase in weight of lower leg from 5 to $15 \mathrm{~kg}$ at constant distance from the pivot to insertion 3 $\mathrm{cm}$, the magnitude of force gets increased from about 3.60 to $4.79 \mathrm{kN}$ [Fig. 5(b)].

The force experienced by hamstring muscle during concentric motion of leg curl as the function of length of the lower leg and weight suspended on machine at constant weight of lower leg $W_{l}=15 \mathrm{~kg}$ is shown in the Fig. 6(a). It is found that the weight suspended on machine has significant effect on the force experienced by the hamstring muscle during leg curl. As the weight suspended 
on the machine is increased, an athlete has to apply more force in the upward direction and hence the force also increases on the hamstring. From Fig. 6(b), it is seen that the magnitude of force decreases with the increase in distance of pivot point from the insertion for all the cases. The magnitude of force experienced by athlete increases with the increase in weight suspended on the machine. With the increase in weight suspended on machine from 10 to $40 \mathrm{~kg}$ at constant distance from the pivot to insertion $3 \mathrm{~cm}$, the magnitude of force increases from about 3.75 to $9.80 \mathrm{kN}$. This signifies that the force on the hamstring muscle during leg curl not only depends upon the external factors but also depends upon the internal structure and formation of muscles.
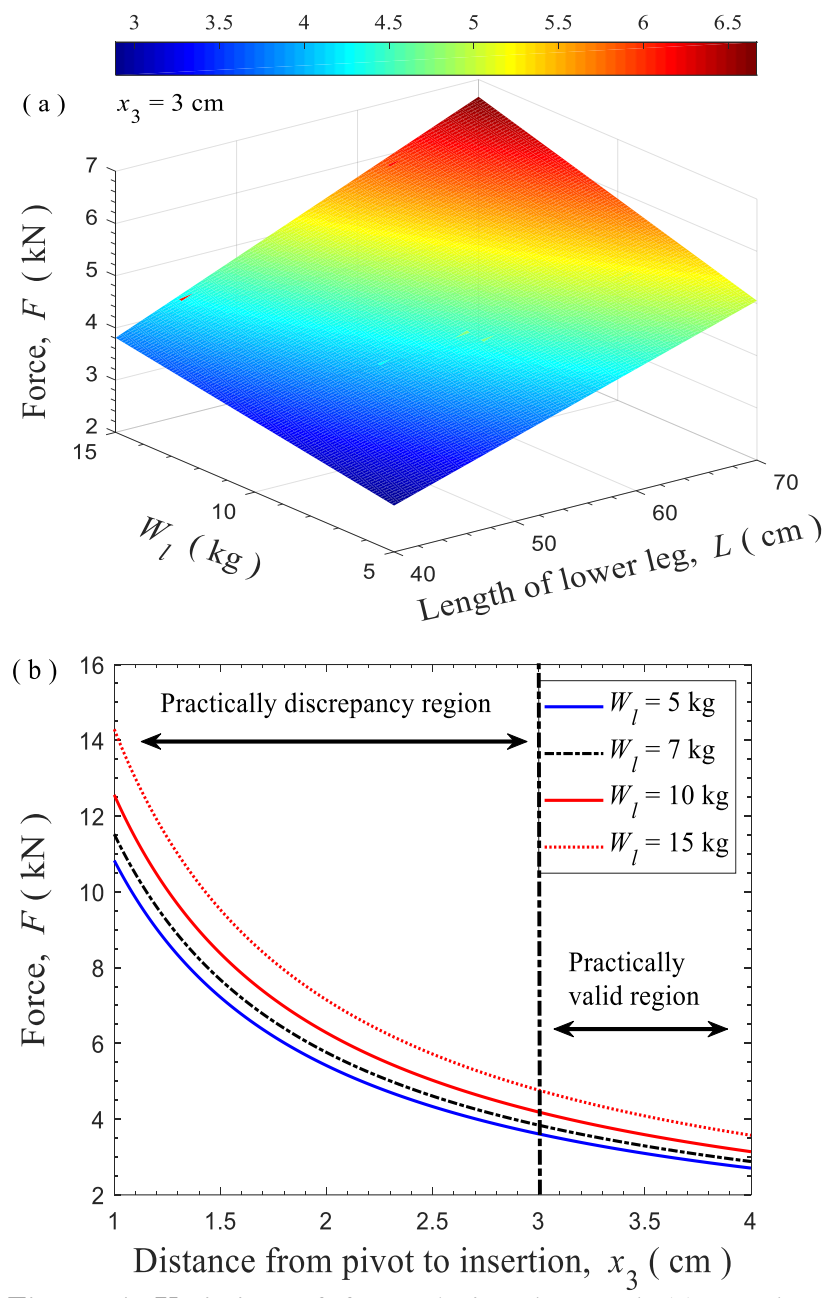

Figure 5. Variation of force during leg curl (a) as the function of length of lower and weight of lower leg and (b) as the function of distance of insertion from the pivot.

The force experienced by the hamstring muscle during concentric motion as the function of distance from pivot to insertion for the different values of length of lower leg is depicted in the Fig. 7. It can be seen that the magnitude of force decreases with the increase in distance of insertion around tendon from the pivot point; however, the magnitude of force increases with the increase in length of lower leg. At constant distance of insertion from pivot 1 $\mathrm{cm}$, the magnitude of force gets increased from about 11.43 to $20.01 \mathrm{kN}$, which is lied in the practically discrepancy region. It is found that when the length of lower leg increases from 40 to $70 \mathrm{~cm}$ at constant distance from pivot to insertion $3 \mathrm{~cm}$, the magnitude of force increases from about 3.81 to $6.67 \mathrm{kN}$. This variation of magnitude of force is lying in the practically valid region.
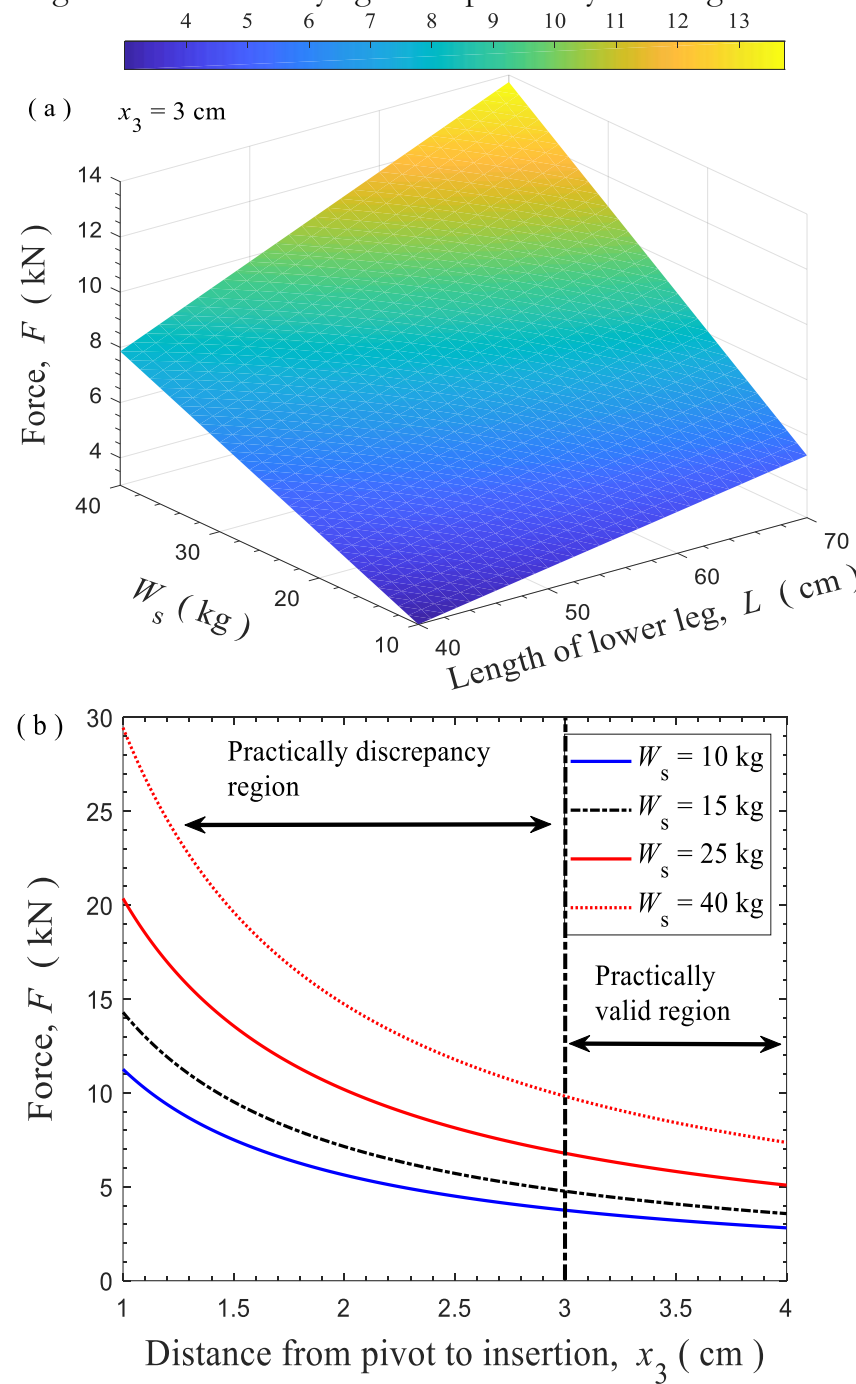

Figure 6. Variation of force on hamstring as the function of (a) weight suspended on the machine and the length of lower leg and (b) distance insertion from the pivot.

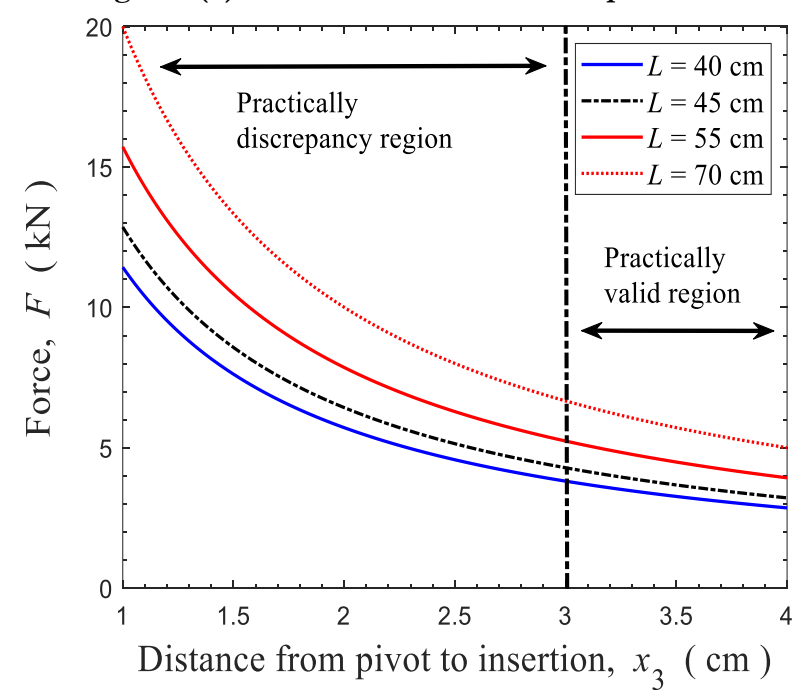

Figure 7. Variation of force on hamstring as the function of insertion around tendon for different lengths of lower leg. 
The force experienced by athletes during concentric motion depends on the size of the upper leg and its variation is depicted in Fig. 8. The upper leg dimension has measurable effect on the force experienced by hamstring during the leg curl. The magnitude of force decreases with the increase in upper leg dimension. With the increase in upper leg dimension $\phi$ from about $30^{\circ}$ to $65^{\circ}$, the magnitude of force decreases from about 4.76 to $2.63 \mathrm{kN}$. The size of the upper leg is different for the different people. It is evident that the people with larger upper leg have hamstring muscle located farther from the line of action of femur than the people with smaller upper leg. This implies that the people with larger upper leg dimensions need to apply less effort to lift the constant weight suspended on the machine. When the dimension of upper leg gets increased, the increased vertical component of force results in decrease of force with the increase in $\phi$.

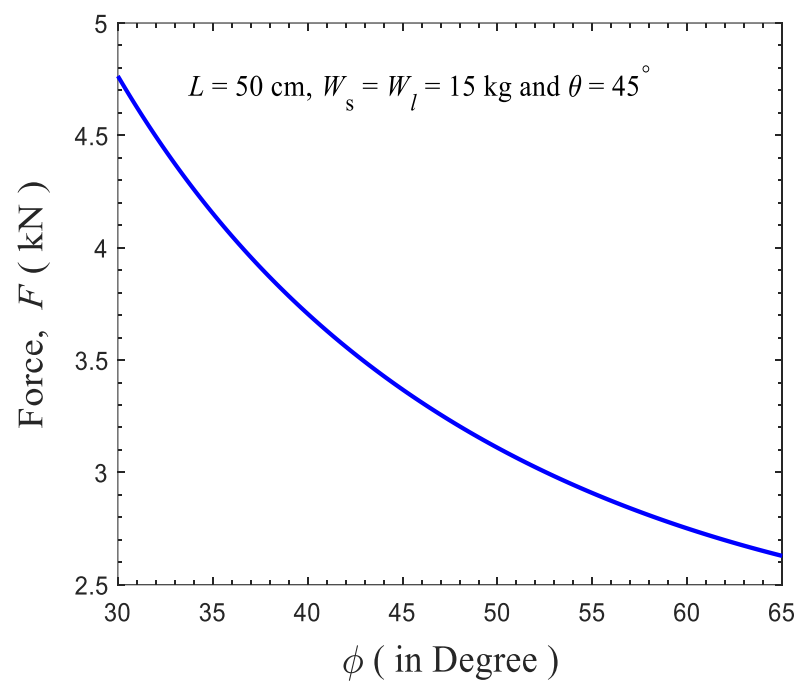

Fig. 8: Variation of force on hamstring as the function of upper leg dimensions.

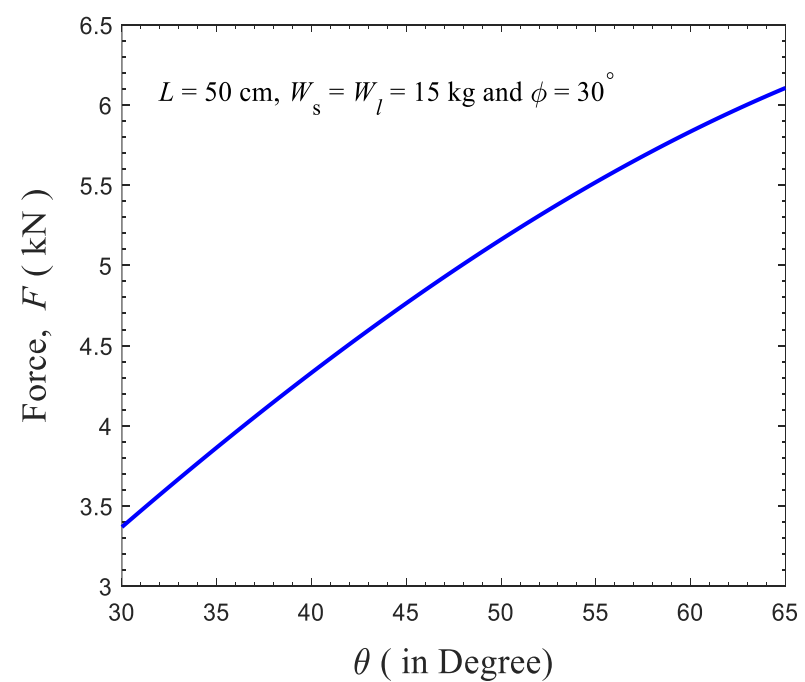

Figure 9. Variation of force on hamstring as the function of angle of suspension with vertical.

The force experienced by athletes during hamstring muscle contraction of leg curl as a function of size of the upper leg is shown in the Fig. 9. It can be seen that the force on the hamstring increases with the increase in angle of suspension with the vertical. The magnitude of force increases from about 3.37 to $6.11 \mathrm{kN}$ for the increase in angle of suspension from $30^{\circ}$ to $65^{\circ}$. The distance from negative $y$-axis to the center of mass of lower leg and the padded roller both increases with the increase in angle between the suspended padded roller and negative y-axis. As a result, moment arm about knee increases which causes to increase the magnitude of force linearly.

\section{CONCLUSIONS}

Leg curl is extensively used by athletes to train hamstring muscle. The mathematical formulation of force experienced, and work done during the eccentric and concentric motion of hamstring has been derived and graphically illustrated. However, the mathematical expression for concentric motion is sophisticated and requires significant study. The force experienced by athletes during concentric motion is presented as the function of length of lower leg, insertion of hamstring around tendon, length of lower leg, weight suspended on the machine, weight of lower leg and upper leg dimensions. It is found that the weight suspended on the machine has more significant effect on the force than the weight of lower leg. For the increase in weight suspended on the machine from 10 to $40 \mathrm{~kg}$ at constant distance from the pivot to insertion $3 \mathrm{~cm}$, the magnitude of force increases from about 3.75 to $9.80 \mathrm{kN}$. This increment of force lies in practically valid region. In the practically valid region $(3$ to $4 \mathrm{~cm}$ ), the magnitude of force increases from about 3.81 to $6.67 \mathrm{kN}$ when the length of lower leg increases from 40 to $70 \mathrm{~cm}$. The insertion of hamstring around the tendon does not change significantly from people to people and the region with distance from pivot to insertion 3 to $4 \mathrm{~cm}$ can be taken as practically valid region. Therefore, it is impossible to measure such high magnitude of force for the different people solely because of insertion cannot be closed to the pivot. With the increase in $\phi$ from about $30^{\circ}$ to $65^{\circ}$, the magnitude of force decreases from about 4.76 to $2.63 \mathrm{kN}$, whereas the magnitude of force increases linearly from about 3.37 to $6.11 \mathrm{kN}$ for the same increment of angle of suspension.

\section{AUTHOR CONTRIBUTIONS}

SBT worked out the theoretical background to address did the calculations and prepared first draft of the manuscript. SB formulated the problem, suggested numerical tools, checked all results, plots (including concept of practical discrepancy and validity in plots 5,6 and 7), and revised the draft manuscript. RK visualized the problem, conceptualized the work finalized the manuscript before submission.

\section{CONFLICT OF INTEREST}

The authors declare no conflict of interest.

\section{DATA AVAILABILITY STATEMENT}

The data that support the findings of this study are available from the corresponding author, upon reasonable request. 


\section{REFERENCES}

Aagaard, P., Simonsen, E.B., Magnusson, S.P., Larsson, B., \& Dyhre-Poulsen, P. (1998). A new concept for isokinetic hamstring: quadriceps muscle strength ratio. The American Journal of Sports Medicine, 26(2), 231-237.

Bigland, B., \& Lippold, O.C.J. (1954). The relation between force, velocity and integrated electrical activity in human muscles. The Journal of Physiology, 123, 214-224.

Christ, S., \& Knortz, K. (1981). Kinesiology corner: The hamstring curl. Strength \& Conditioning Journal, 3(6), 52-53.

Croisier, J.L., Forthomme, B., Namurois, M.H., Vanderthommen, M., \& Crielaard, J.M. (2002). Hamstring muscle strain recurrence and strength performance disorders. The American Journal of Sports Medicine, 30(2), 199-203.

Faulkner, J.A. (2003). Terminology for contractions of muscles during shortening, while isometric, and during lengthening. Journal of Applied Physiology, 95(2), 455-459.

Floyd, R.T., \& Thompson C.W. (2015). Manual of structural kinesiology, 19th Edition, USA: McGraw Hill, New York.

Grassi, C.A., Fruheling, V.M., Abdo, J.C., de Moura, M.F.A., Namba, M., da Silva, J.L.V., da Cunha, N.A.M., de Oliveira Franco, A.P.G., Costa, I.Z., \& Filho E.S. (2013). Hamstring tendons insertion-an anatomical study. Revista Brasileira de Ortopedia, 48, 417-420.

Hill, A.V. (1925). Length of muscle, and the heat and tension developed in an isometric contraction. The Journal of Physiology, 60, 237-263.

Hody, S., Croisier, J.L., Bury, T., Rogister, B., \& Leprince, P. (2019). Eccentric muscle contractions: risks and benefits. Frontiers in Physiology, 10, 536. doi: 10.3389/fphys.2019.00536.

Kilgallon, M., Donnelly, A.E., \& Shafat, A. (2007). Progressive resistance training temporarily alters hamstring torque-angle relationship. Scandinavian Journal of Medicine \& Science in Sports, 17(1), 18-24.
Kjaer, M., \& Heinemeier, K.M. (2014). Eccentric exercise: acute and chronic effects on healthy and diseased tendons. Journal of Applied Physiology, 116(11), 1435-1438.

Ling, S.J., Moebs, W., \& Sanny, J. (2016). University Physics, Volume 2. USA: OpenStax Rice University, Houston, Texas.

Maganaris, C.N. (2001). Force-length characteristics of in vivo human skeletal muscle. Acta Physiologica Scandinavica, 172(4), 279-285.

Milošević, B.M., Blagojević, M., Pilipović, S., \& Tošić, B. (2000). The muscle contraction and the force production. Proceedings of the XVIII International Symposium of Biomechanics in Sport, pp. 183-186.

Mjølsnes, R., Arnason, A., Østhagen, T., Raastad, T., \& Bahr, R. (2004). A 10-week randomized trial comparing eccentric vs. concentric hamstring strength training in well-trained soccer players. Scandinavian Journal of Medicine \& Science in Sports, 14(5), 311-317.

Padulo, J., Laffaye, G., Ardigò, L.P., \& Chamari, K. (2013). Concentric and eccentric: muscle contraction or exercise Journal of Human Kinetics, 37, 5-6.

Vogt, M., \& Hoppeler, H.H. (2014). Eccentric exercise: mechanisms and effects when used as training regime or training adjunct. Journal of Applied Physiology. 116, 1446-1454.

Wright, G.A., Delong, T.H., \& Gehlsen, G. (1999). Electromyographic activity of the hamstrings during performance of the leg curls, stiff-leg deadlift, and back squat movements. The Journal of Strength \& Conditioning Research, 13(2), 168-174.

\section{Internet source}

https://robinsonsstrengthandendurancecoaching.wordpress.com /2018/01/12/leg-strength-training 